$\underline{\text { Supporting Information }}$

\title{
Particle-Rod Hybrids: Growth of Arachidic Acid Molecular Rods from Capped Cadmium Selenide Nanoparticles
}

\author{
Dongzhong Chen, Ruomiao Wang, Indika Arachchige, \\ Guangzhao Mao*, Stephanie L. Brock
}

\section{Experimental Section.}

\section{CdSe nanoparticle synthesis and capping}

\section{Materials}

Trioctylphosphineoxide (TOPO, 90\%), CdO (99.99\%), Se powder (99.5\%), trioctylphosphine (TOP, 90\%), 11-mercaptoundecanoic acid (MUA, 95\%) and tetramethylammonium hydroxide pentahydrate (TMAH, 97\%) are purchased from Aldrich. Ethyl acetate and ethyl ether are purchased from Fisher and ntetradecylphosphonic acid (TDPA, 98\%) is purchased from Alfa aesar. All chemicals are used as received unless specified.

\section{Synthesis}

The high-temperature CdSe nanoparticle synthesis is adapted from Peng et al (J. Am. Chem. Soc. 2001, 123, 183). In a typical synthesis of CdSe nanocrystals, $0.0514 \mathrm{~g}$ of $\mathrm{CdO}, 0.1116 \mathrm{~g}$ of TDPA and $3.7768 \mathrm{~g}$ of TOPO is slowly heated to $300-320{ }^{\circ} \mathrm{C}$ under $\mathrm{Ar}$ flow. At about $300{ }^{\circ} \mathrm{C}$, the reddish brown $\mathrm{CdO}$ powder dissolves in the TOPO and TDPA mixture to form a homogeneous colorless solution. The temperature of the solution is cooled to $270{ }^{\circ} \mathrm{C}$, and the selenium stock solution $(0.0314 \mathrm{~g}$ of Se powder in $2.4 \mathrm{~mL}$ of TOP) is quickly injected. The resultant CdSe nanocrystals are grown at $250{ }^{\circ} \mathrm{C}$ for $4 \mathrm{~h}$ and precipitated with methanol.

\section{Thiol coating of CdSe nanoparticles by MUA}

Thiol coating of CdSe nanocrystals is adapted from Aldana et al (J. Am. Chem. Soc. 2001, 123, 8844) using varying ratios of CdSe:MUA (1:3, 1:5, and 1:10). An appropriate amount (e.g., $0.4672 \mathrm{~g}$ of MUA, if CdSe:MUA ratio is 1:5) of MUA is dissolved in 15 
$\mathrm{mL}$ of methanol and the $\mathrm{pH}$ of the resulting solution is adjusted to $>10$ with tetramethylammonium hydroxide pentahydrate. In the absence of light, the methanolprecipitated $\mathrm{CdSe}$ nanocrystals are dissolved in the above mixture and the resulting solution is stirred under Ar flow for $2 \mathrm{~h}$. Ethyl acetate and ethyl ether are used to precipitate and wash (2-3 times) the MUA-capped nanocrystals, which are subsequently dispersed in isopropanol to form the CdSe sol.

\section{Characterization of nanoparticles}

TEM analyses are conducted in bright-field mode using a JEOL FastEM 2010 HR TEM electron microscope with $\mathrm{LaB}_{6}$ thermoelectric emission gun working at $200 \mathrm{kV}$. As-prepared nanocrystals are dissolved in toluene (CdSe) or methanol (CdSe-MUA), placed on a 200-mesh copper carbon grid and the solvent is dried by evaporation. SEM images are obtained using a Hitachi S-2400 microscope at $25 \mathrm{keV}$ in secondary electron mode. Vacuum dried (under dark conditions) CdSe-MUA nanocrystals are spread on carbon adhesive tabs on an aluminum stub. An in situ EDAX unit (EDAX Inc. PV 9900) attached to the SEM is used to determine the elemental composition of the CdSe-MUA nanoparticles (Table 1).

\section{Spin coating of $\mathrm{AA}$ and MUA-CdSe films and AFM characterization}

Stock solutions of AA (>99\%, Sigma-Aldrich) and MUA-CdSe nanoparticles in isopropanol (Fisher Scientific, spectranalyzed) are mixed to yield $0.1 \mathrm{mM}$ AA and 0.1 mM MUA-CdSe. $100 \mu \mathrm{l}$ of the mixed solution is dispensed onto a freshly cleaved HOPG surface spinning at $3000 \mathrm{rpm}$ for 60 seconds. The spin-coated samples are imaged using a Dimension 3100 AFM (Veeco Metrology). Height, amplitude, and phase images are obtained in Tapping Mode in ambient air with silicon tips (TESP, Veeco) using a scan rate of $1 \mathrm{~Hz}$. Integral and proportional gains are approximately 0.3 and 0.5 respectively. Only flattened height images are shown. The films are usually imaged immediately after film preparation. However, the same particle-rod hybrid structure can be obtained at least two weeks after film preparation and storage at room temperature. The crystal structure of stearic acid is constructed with the Materials Studio software program (Accelrys). 


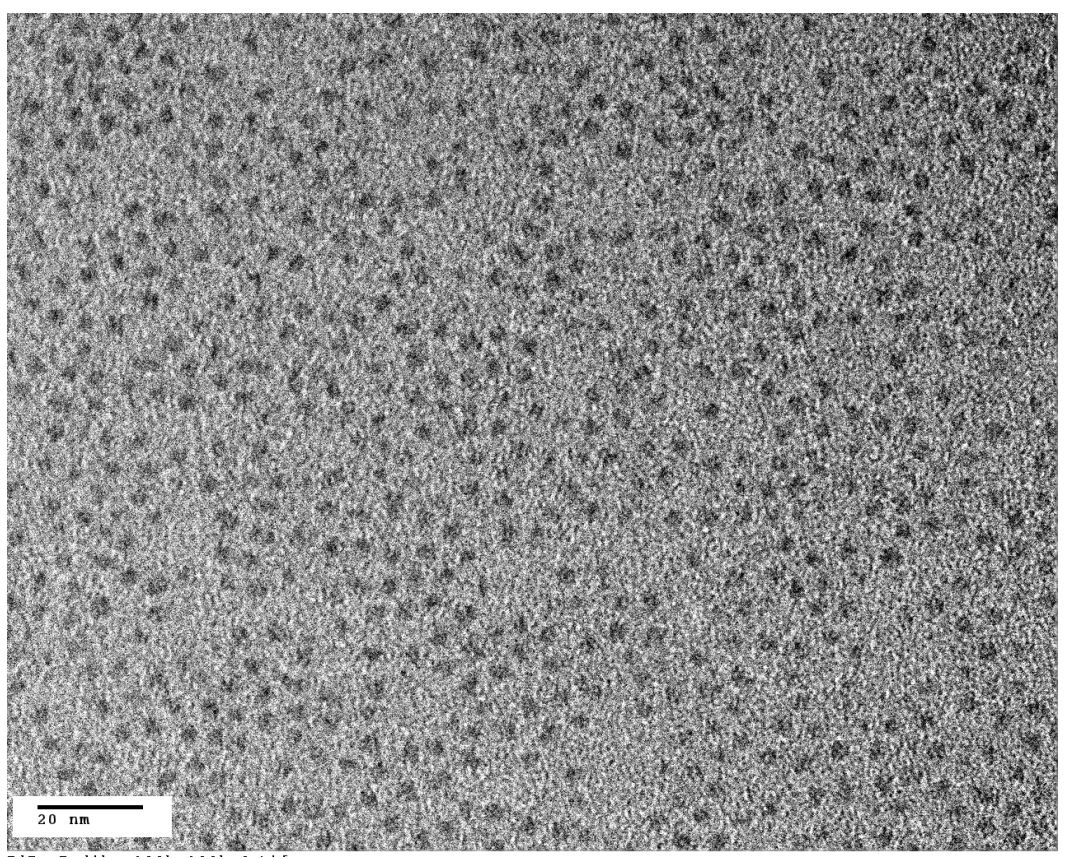

Figure 1. TEM image of CdSe nanoparticles before capping with MUA. CdSe nanoparticle size: $2.97 \pm 0.59 \mathrm{~nm}$.

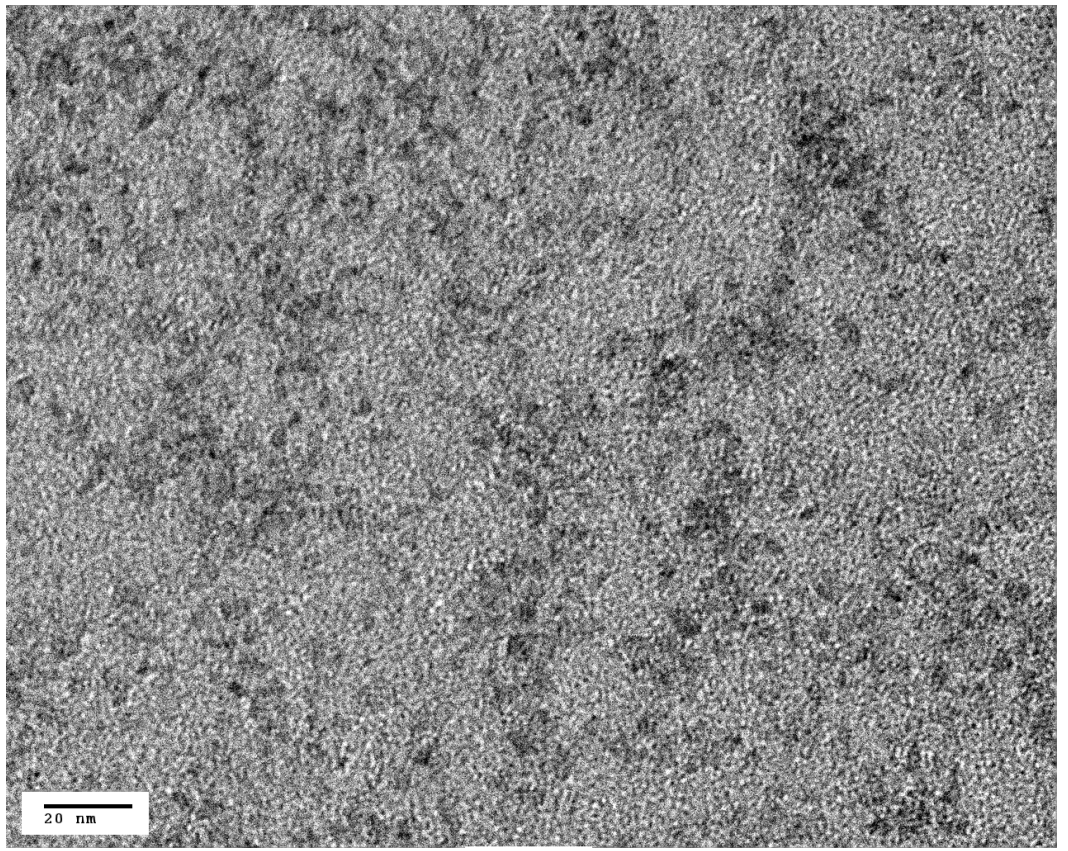

Figure 2. TEM image of MUA-capped CdSe nanoparticles showing aggregate formation. 


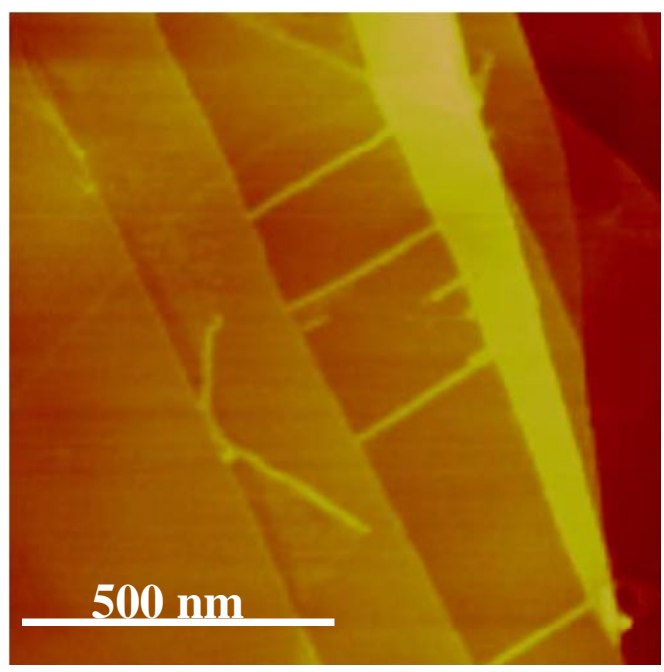

Figure 3. An AFM height image showing AA molecular rods nucleated at the defect lines on the same substrate where rods of identical size also emanating from the MUACdSe nanoparticles (MUA: CdSe $=0.50: 1$ ). The sample is spin coated from a $0.1 \mathrm{mM}$ AA and $0.1 \mathrm{mM}$ MUA-CdSe isopropanol solution on HOPG. Z-range $=8 \mathrm{~nm}$.

Table 1. The chemical composition of MUA-CdSe by SEM/EDAX analysis.

\begin{tabular}{|c|c|c|}
\hline CdSe : MUA synthesis ratio & $\mathrm{Cd}: \mathrm{Se}$ & observed \\
\hline $1: 3$ & $1.00: 1.04$ & 0.38 \\
\hline $1: 5$ & $1.00: 1.20$ & $: 0.50$ \\
\hline $1: 10$ & $1.00: 1.03$ & $: 0.68$ \\
\hline
\end{tabular}

